Research Article

\title{
Characteristics of Orbit Determination with Short-Arc Observation by an Optical Tracking Network, OWL-Net
}

\author{
Jin Choi, ${ }^{1,2}$ Jung Hyun Jo $\mathbb{D}^{1},{ }^{1,2}$ Kyung-Min Roh, ${ }^{1}$ Hong-Suh Yim, ${ }^{1}$ Eun-Jung Choi, \\ and Sungki Cho ${ }^{1}$ \\ ${ }^{1}$ Korea Astronomy and Space Science Institute, Daejeon 34055, Republic of Korea \\ ${ }^{2}$ University of Science and Technology, Daejeon 34113, Republic of Korea \\ Correspondence should be addressed to Jung Hyun Jo; jhjo39@kasi.re.kr
}

Received 8 April 2018; Accepted 11 June 2018; Published 18 July 2018

Academic Editor: Yue Wang

Copyright ( 2018 Jin Choi et al. This is an open access article distributed under the Creative Commons Attribution License, which permits unrestricted use, distribution, and reproduction in any medium, provided the original work is properly cited.

\begin{abstract}
An optical tracking network, the Optical Wide-field patroL Network (OWL-Net), has been developed to maintain the orbital ephemeris of 11 domestic low Earth orbit satellites. The schedule overlapped events were occurred in the scheduling of the OWL-Net with reduction of the optical observation chances. A short-arc observation strategy for the OWL-Net was tested to reduce schedule overlapped events with the optical observation simulation and the orbit determination. In the full-scale optical observation simulation from January 2014 to December 2016, the most frequent overlapped events were occurred 127, 132, and 116 times in the 4th, 34th, and 18th weeks of 2014, 2015, and 2016, respectively. The average number of overlapped event for three years was over $10 \%$ for the whole observation chances of five stations. Consequently, the short-arc observation strategy reduced the schedule overlapped events for every observation target of the OWL-Net. In case of the $5 \mathrm{~s}$ and $10 \mathrm{~s}$ cases, the most schedule overlapped events were removed. The test results of the orbit determination results show that the most maximum orbit prediction errors after seven days are maintained at $<10 \mathrm{~km}$ in the in-track direction for the short-arc observation simulations. The results demonstrate that the short-arc optical observation strategy is more optimal to maintaining the accuracy of orbital ephemeris with more observation chances.
\end{abstract}

\section{Introduction}

Space objects can be tracked with various types of equipment [1], and optical tracking devices have been used to collect observation data for space objects from low Earth orbit (LEO) to the geosynchronous orbit (GSO). Among the 43,269 known space objects, 13,393 objects were registered as low Earth orbit (LEO) objects as of 6 April 2018. LEO is defined as a volume of space that has the altitude from 0 to $2000 \mathrm{~km}$ by the Inter-Agency Space Debris Coordination Committee (IADC) Space Debris Mitigation Guidelines [2]. The Baker-Nunn camera was an early type of optical tracking network installed at 11 sites around the world. The primary goal of the Baker-Nunn camera was to track launched vehicles and satellites with a wide field of view [3]. Ground-Based Electro-Optical Deep Space Surveillance (GEODSS) was another optical tracking network. GEODSS replaced the Baker-Nunn camera in 1979 [4]. The United States operates these facilities and also provides a catalogue of unclassified space objects publicly via the website Space-Track.

The Korean Astronomy and Space Science Institute (KASI) has been developing an optical tracking network called the Optical Wide-field patroL Network (OWL-Net) since 2010. KASI plans to install five optical tracking systems in Mongolia, Morocco, Israel (at the Wise observatory), the United States (at the Mt. Lemmon Astronomical Observatory (LOAO)), and South Korea (at the Bohyun Astronomical Observatory (BOAO)). The main observation targets of the OWL-Net are 11 South Korean LEO satellites shown in Figure 1. Monitoring the South Korean geostationary Earth orbit (GEO) region near 128 degrees of longitude is another goal of the OWL-Net. The candidate sites were selected in the light of results for an optical tracking 


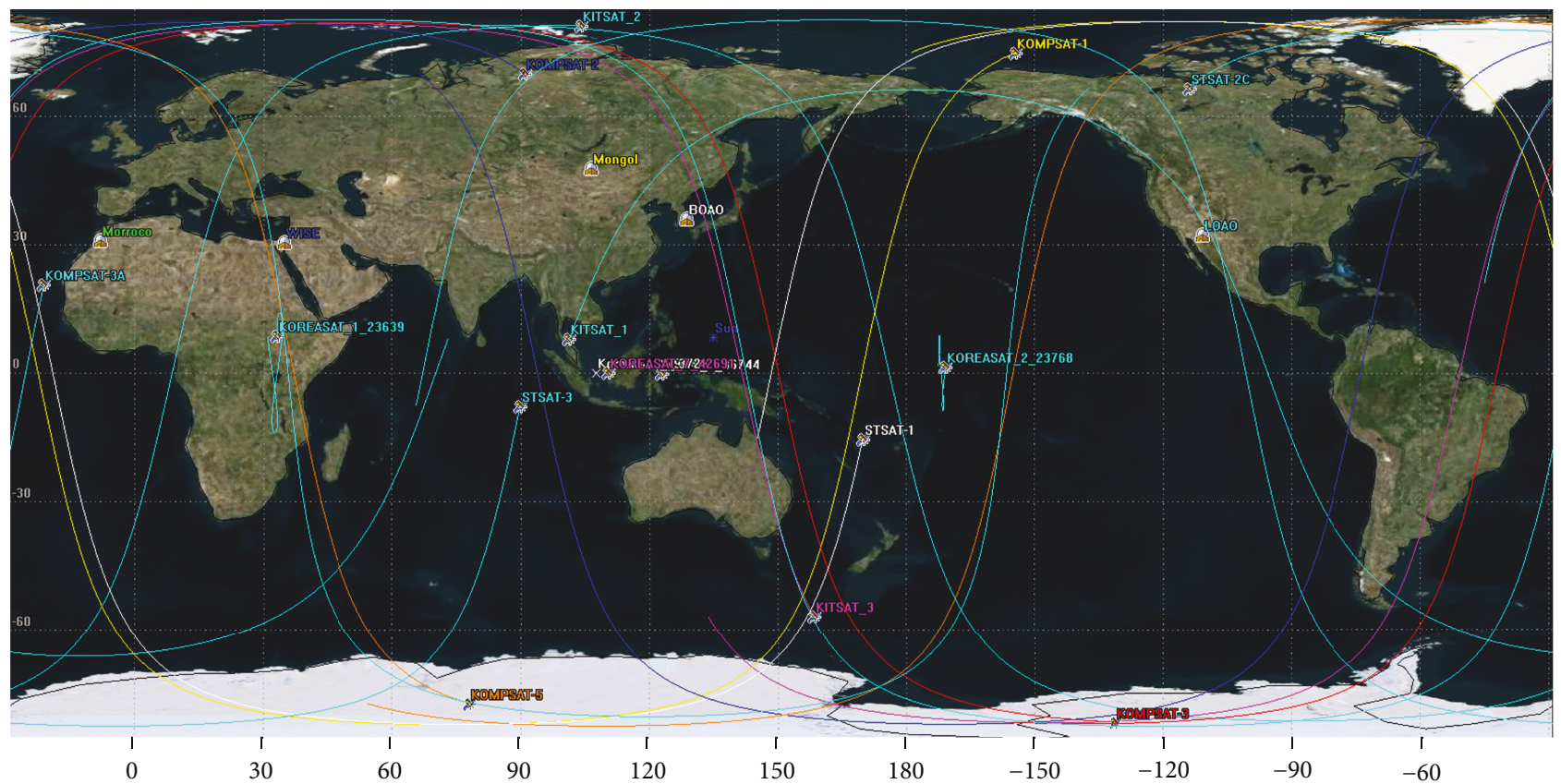

FIGURE 1: OWL-Net site locations and observation target, 11 LEO satellites and 6 GEO satellites (4 GEOs are located near 128 E of longitude; Koreasat_1 and 2 are drifting eastward).

simulation with 11 observation targets of LEO satellites [5]. The OWL-Net can make an observation with a chopper to obtain dozens of measurements per shot [6,7]. The authors have performed test observations and orbit determination research for various satellites [8-11]. OWL-Net headquarters create daily schedules [based on two-line element (TLE) data from the Joint Space Operations Center (JSpOC)] for automatic observation of scheduled targets.

Occasionally, two or more targets will be visible to the OWL-Net at the same time (overlapped tracks). The optical tracking equipment is a passive observation system, and the observation time of LEO observation targets is limited to terminator conditions at dawn and dusk (when the sky is dark but the target remain sun-illuminated). When the target visibility of multiple targets is overlapped, only one target can be tracked at a time. When this happens, the number of observations for nonselected targets is reduced. However, too sparse or irregular observation data can affect the quality of orbit determination [12]. We designated this problem as an optical observation overlapping problem.

Observation conditions such as frequency of track and arc length can affect the orbit estimation accuracy. Vallado and Carter showed that highly accurate orbit determination is possible with dense observation data for $120 \mathrm{~s}$ (a few hundred observations per satellite pass) [13]. Bennett et al. showed that short-arc observations for $5 \mathrm{~s}$ from two passes are sufficient to maintain the orbital elements for the catalogue [14]. Park et al. analysed the minimum amount of data for observed LEO satellites by using the OWL-Net [9]. In this analysis, the orbit determination for the various observation scenarios with the same arc-length were analysed to find the optimal observation condition. In the case of
GEO objects, the relation between the orbit determination accuracy and arc length was analysed theoretically. Analysis was verified again with real observation data [15]. Tombasco and Axelrad also showed the relation between orbit determination accuracy and observation frequency for the Galaxy 15 GEO satellite with tracking and data relay satellite (TDRS) data [16]. The arc length was the dominant factor for orbit determination accuracy of GEO objects. However, more frequently observed data also improved orbit determination accuracy. Orbit determination accuracy with the angleonly data case was also analysed for sparse, sporadic, and dense cases using a single optical telescope [11]. The orbit determination results showed that the arc length was the dominant factor for angle-only orbit determination of GEO satellites. However, even in GEO satellite orbit determination tests, multiarc observation cases have shown much more accurate orbit determination results than the single arc observation case.

In this paper, we analyse the effectiveness of a short-arc optical observation strategy for orbital ephemeris maintenance using the OWL-Net. Firstly, the orbital characteristics of 11 LEO satellites and observation simulation statistics of the OWL-Net were analysed using consecutive TLEs. Secondly, we simulated weekly overlapped observations for 2014, 2015, and 2016. Thirdly, an orbit determination test was done with short-arc observation strategy. Generally, a short-arc optical observation strategy can allow for more frequent observation opportunities. We have attempted to verify various short-arc strategies for effective observation scheduling. Finally, the orbit estimation test for the shortarc optical observation strategy was performed using the real observation data from the OWL-Net. 
TABLE 1: Orbital characteristics of 11 Korean LEO satellites on 6 April 2018.

\begin{tabular}{|c|c|c|c|c|c|c|c|c|}
\hline NORAD ID & Satellite name & International designation & Period & Inclination & Apogee & Perigee & Designed Orbit & Operational \\
\hline 22077 & OSCAR 23 (KITSAT-1) & 1992-052B & 111.93 & 66.08 & 1319 & 1312 & LEO & no \\
\hline 22825 & KITSAT-B & 1993-061C & 100.7 & 98.78 & 797 & 786 & LEO & no \\
\hline 25756 & KITSAT-3 & 1999-029A & 99.06 & 98.41 & 723 & 705 & LEO & no \\
\hline 26032 & KOMPSAT-1 & 1999-070A & 98.03 & 97.84 & 667 & 662 & $\mathrm{SSO}$ & no \\
\hline 27945 & STSAT- 1 & $2003-042 G$ & 98.31 & 97.91 & 686 & 670 & SSO & no \\
\hline 29268 & KOMPSAT-2 & 2006-031A & 98.48 & 98.12 & 699 & 673 & $\mathrm{SSO}$ & no \\
\hline 38338 & KOMPSAT-3 & 2012-025B & 98.53 & 98.17 & 695 & 682 & SSO & yes \\
\hline 39068 & STSAT-2C & $2013-003 \mathrm{~A}$ & 95.94 & 80.23 & 852 & 276 & SSO & no \\
\hline 39227 & KOMPSAT-5 & $2013-042 \mathrm{~A}$ & 95.71 & 97.6 & 554 & 552 & $\mathrm{SSO}$ & yes \\
\hline 39422 & STSAT-3 & 2013-066G & 96.69 & 97.69 & 615 & 585 & SSO & yes \\
\hline 40536 & KOMPSAT-3A & $2015-014 \mathrm{~A}$ & 95.22 & 97.57 & 537 & 521 & SSO & yes \\
\hline
\end{tabular}

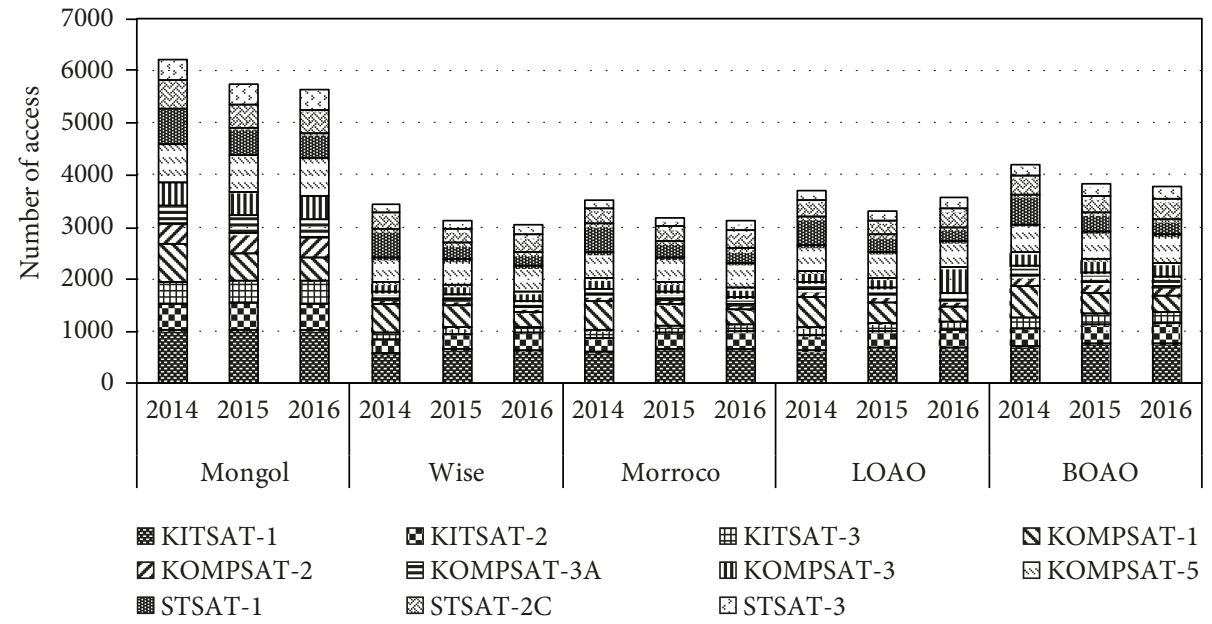

FIgURE 2: Number of accesses for the 11 LEO observation targets at OWL-Net from 2014 to 2016.

\section{Optical Observation Schedule Overlapping Problem}

2.1. OWL-Net Observation Target and Observation Simulation. The primary observation target of the OWLNet is 11 Korean LEO satellites. Only four LEO satellites were in operation on 6 April 2018. Table 1 shows identification (ID) and orbital characteristics for the 11 LEO satellites. All of LEO satellites (except for STSAT-2C) have circular orbits, with eight in Sun-synchronous orbit (SSO). The SSO satellites revisit the same local position at the same local time with the fixed line of nodes of orbit to the sun [17]. Because optical tracking systems can only make observations near dawn and dusk for LEO satellites, the observation opportunities are very limited for these SSO satellites.

We analysed the orbital characteristics of these satellites to look for the dominant parameters of optical observation overlap events for the ground-based observation. Firstly, the orbital shape can be a dominant parameter; we note that, except for STSAT-2C, the remaining 10 domestic LEO satellites have similar eccentricities. The orbital plane location can be another dominant parameter. The right ascension of the ascending node (RAAN) and the inclination determine the rotation of the orbit from the vernal equinox and degree of tilt of the orbital plane, respectively. Except for KITSAT-1 and STSAT-2C, the inclinations of domestic LEO satellites are similar to each other. If the orbital shape and the inclination are similar and the orbital planes are close longitudinally, the possibility of an optical observation overlap event is increased as these satellites can pass above the observing station at a similar local time. If a ground station is positioned near 30 degrees of latitude, LEO satellites from 500 to $800 \mathrm{~km}$ can be observed at the same time from a single ground station within the orbital plane's maximum longitudinal difference of 40 degrees.

OWL-Net observations were simulated from 2014 to 2016 with optical observation constraints. Figure 2 shows the number of visible passes for the 11 LEOs with the OWL-Net from 2014 to 2016 (from Mongolia, Wise, Morocco, LOAO, and BOAO). Almost all LEOs have similar numbers of observable passes for each site over the three years. The Mongolian site has the most observation opportunities for polar orbiting satellites due to its higher latitude. The BOAO site has the second most observable passes. KITSAT-1 has the largest number of observation opportunities among the 11 satellites. KOMPSAT- 1 and KOMPSAT-5 


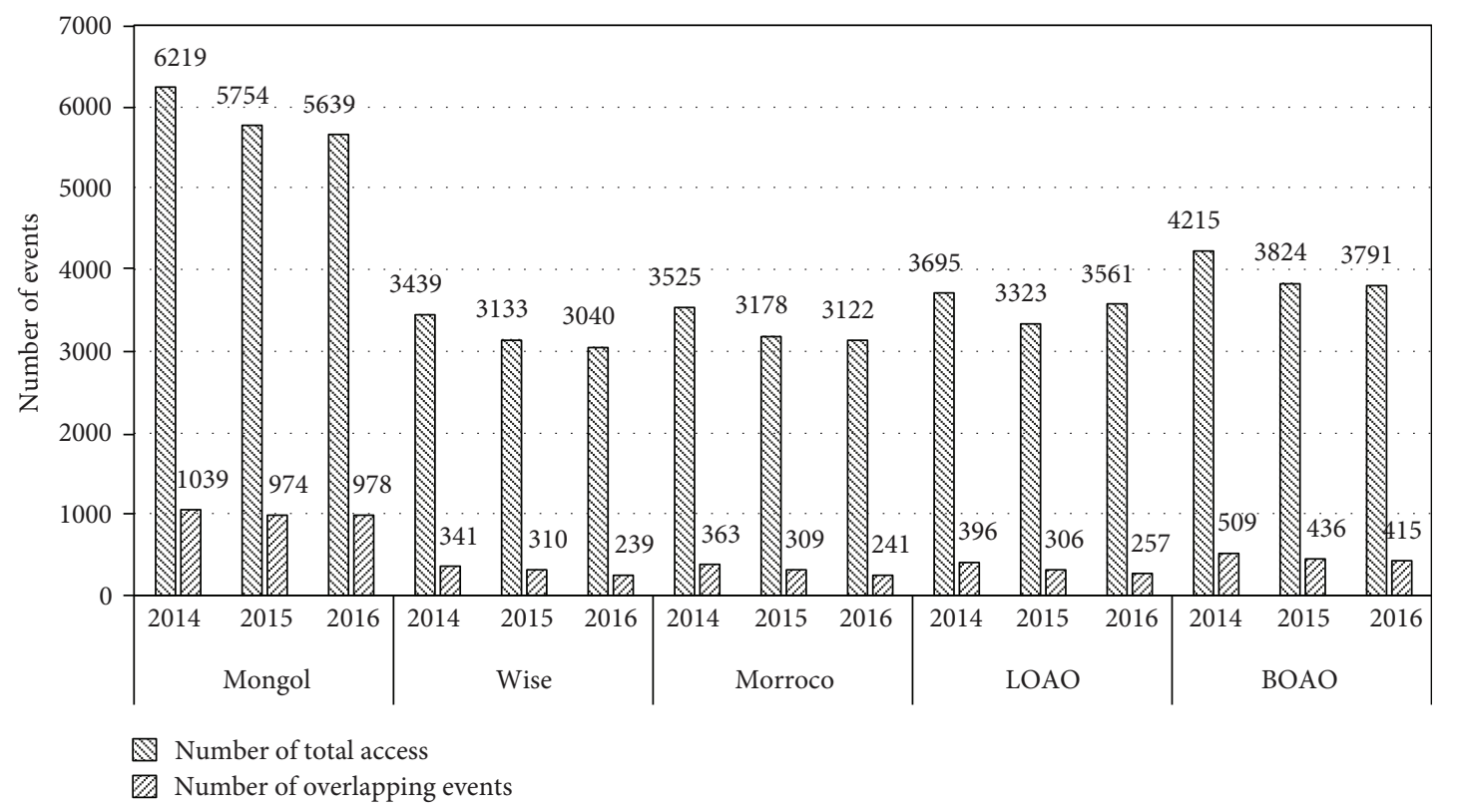

FIgURE 3: Number of total accesses and overlapped events for the 11 LEO observation targets at the OWL-Net from 2014 to 2016.

also have a larger number of observation opportunities than the others. KITSAT-3, KOMPSAT-2, and KOMPSAT-3A have fewer observation opportunities than the others, with $<200$ observation opportunities every year for four sites (excepting the Mongolian site). Weather conditions and the possibility of system anomalies were not considered in this simulation.

\subsection{Statistics of Overlapped Event Simulation of the OWL-} Net. The OWL-Net system was designed to operate an orbit determination process every week. We analysed weekly based overlap events for 2014, 2015, and 2016. TLEs from JSpOC were used for this simulation. We used a general optical tracking condition with -6 degrees as the sun elevation limit and 5 degrees as the site elevation limit.

The number of overlap events for the OWL-Net stations is presented in Figure 3. The number of overlap events schedule comprised almost $10 \%$ of the total number of observable passes for every site during the three years. In 2015, the number of overlap events at the Mongolian site was the highest, with 974 overlapped events. This is $\sim 40 \%$ of the total number of overlapped events for five OWL-Net stations in 2015. For the other four stations, the numbers of overlap events in 2015 were 310 at Wise, 309 at Morocco, 306 at LOAO, and 436 at BOAO. The Mongolian station has a higher latitude than the other four stations. We could not discern any annual variation for the total overlap events for the 11 LEOs at any of the OWL-Net sites. Figure 4 shows the variation of weekly number of overlap events at each OWL-Net site for 2014, 2015, and 2016. This weekly base analysis was undertaken because the OWL-Net system was designed to implement weekly base orbit determination. The Mongolian site still had the most overlap events for the three years as well as the most total available passes. Among the 52 weeks of 2015 , overlap events occur most frequently in the 34 th week (140 events). The 4 th and 18 th weeks experienced the most overlapping events of 2014 and 2016, respectively. However, we also could not discern a weekly tendency of change in the number of overlapped events.

When the overlapped events were occurred, the one observation schedule should be selected among some observation chances. Therefore, the other observation chances were cancelled. Furthermore, the optical observation chance can be reduced by the weather condition and system condition in reality. These too sparse observation conditions can result in failure or bad quality of the orbit determination.

As the number of domestic LEO satellites increases, the frequency of overlapped events will also increase. The overlap problem can be solved either by using more ground stations for covering more observation time or with an effective observation strategy for orbit determination accuracy. However, more ground stations are not an option due to the higher costs. The short-arc optical observation strategy could provide a more acceptable and better solution for the optical observation overlap problem.

\section{Orbit Determination Test for Short-Arc Optical Observation Strategy}

3.1. Analysis of the Number of Overlapped Event for Short-Arc Optical Observation Strategy. Various short-arc optical observation strategies were devised with the arc length of each pass. Firstly, we considered Bennett et al.'s work [14]. In this research, the arc length was set as 5 seconds for two passes. We fixed the arc length as $5 \mathrm{~s}$, but the number of passes was not limited. Because the OWL-Net system was designed for weekly orbit determination. Bennett's work focused on the follow-up observation. Therefore, some satellites can have more optical observation opportunities than the others in this study. Secondly, we selected $120 \mathrm{~s}$ from Vallado and Carter's work [13]. Furthermore, we selected 

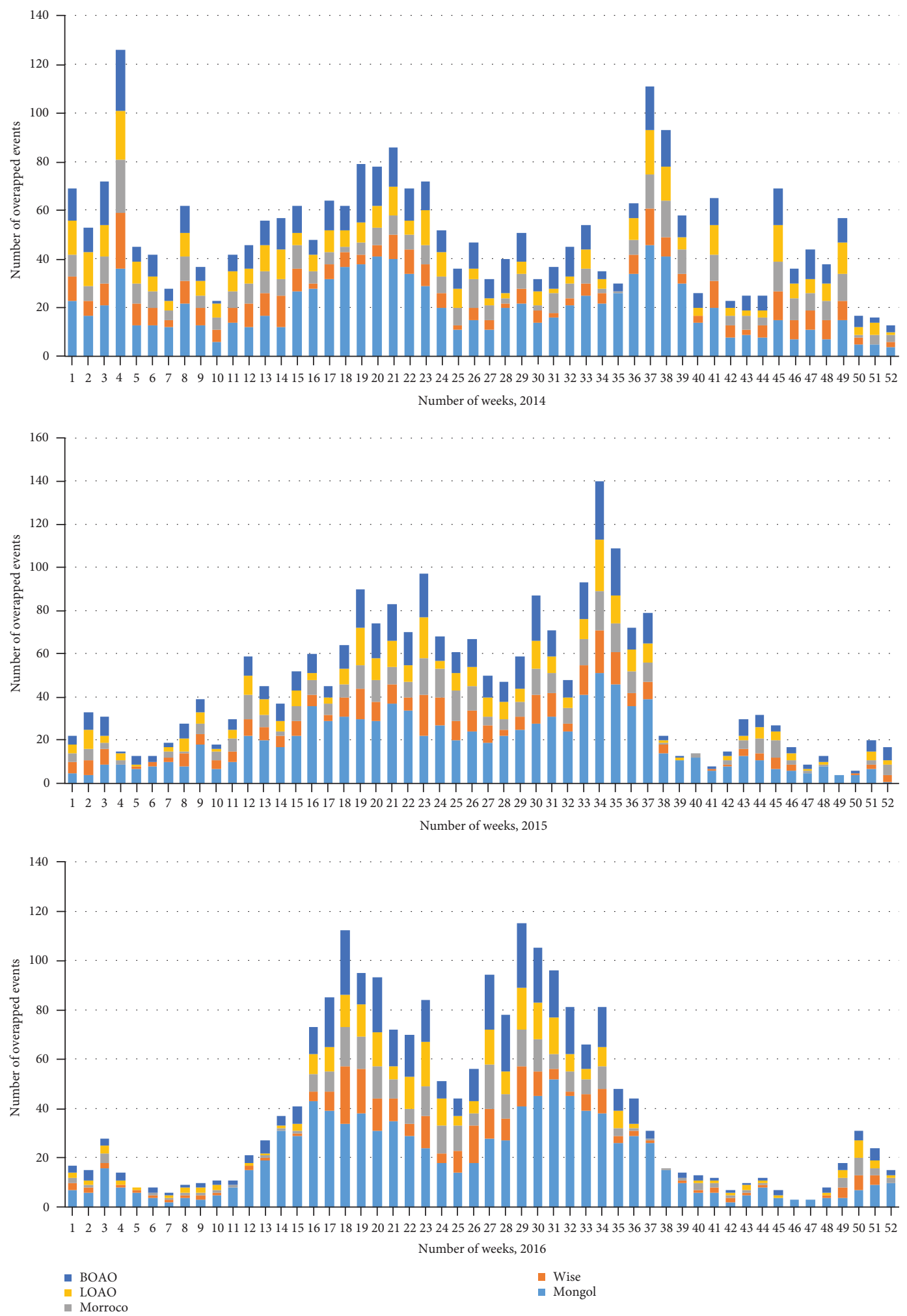

FIGURE 4: Weekly variation of number of optical observation overlapped events for 11 LEO observation targets at the five OWL-Net stations from 2014 to 2016.

$250 \mathrm{~s}$ as a test case. The average observation duration for the 11 domestic LEO satellites at the OWL-Net stations in 2015 was $\sim 250 \mathrm{~s}$. We also selected three other cases $(10 \mathrm{~s}, 60 \mathrm{~s}$, and full arc) to investigate the number of overlap events.
The full arc indicated the maximum observation time duration under the optical observation constraints.

The number of optical observation overlap events is presented in Figure 5. Most overlap events occurred at the 


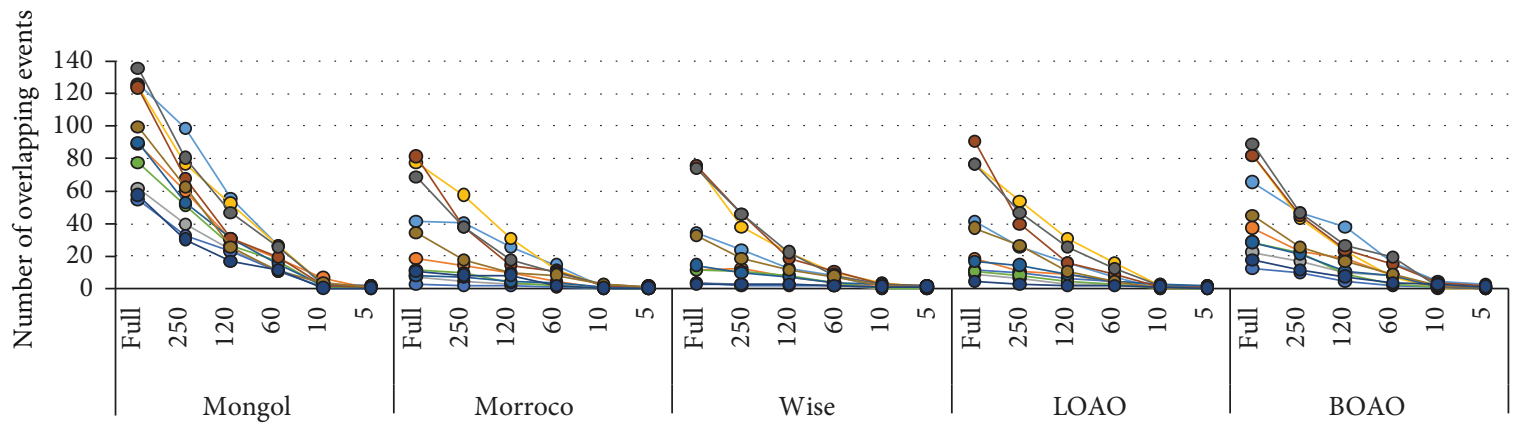

(a)

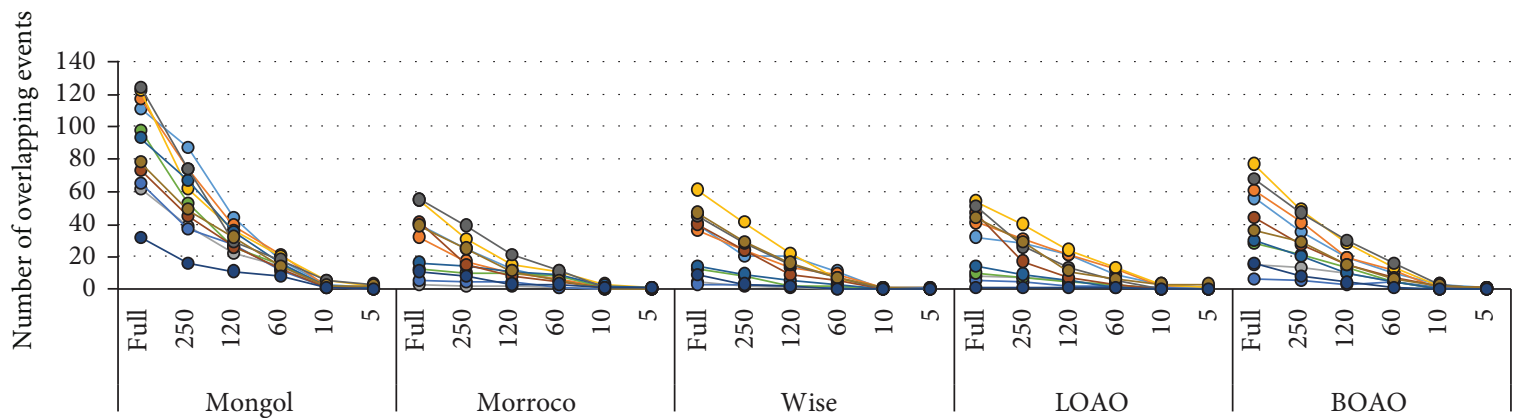

(b)

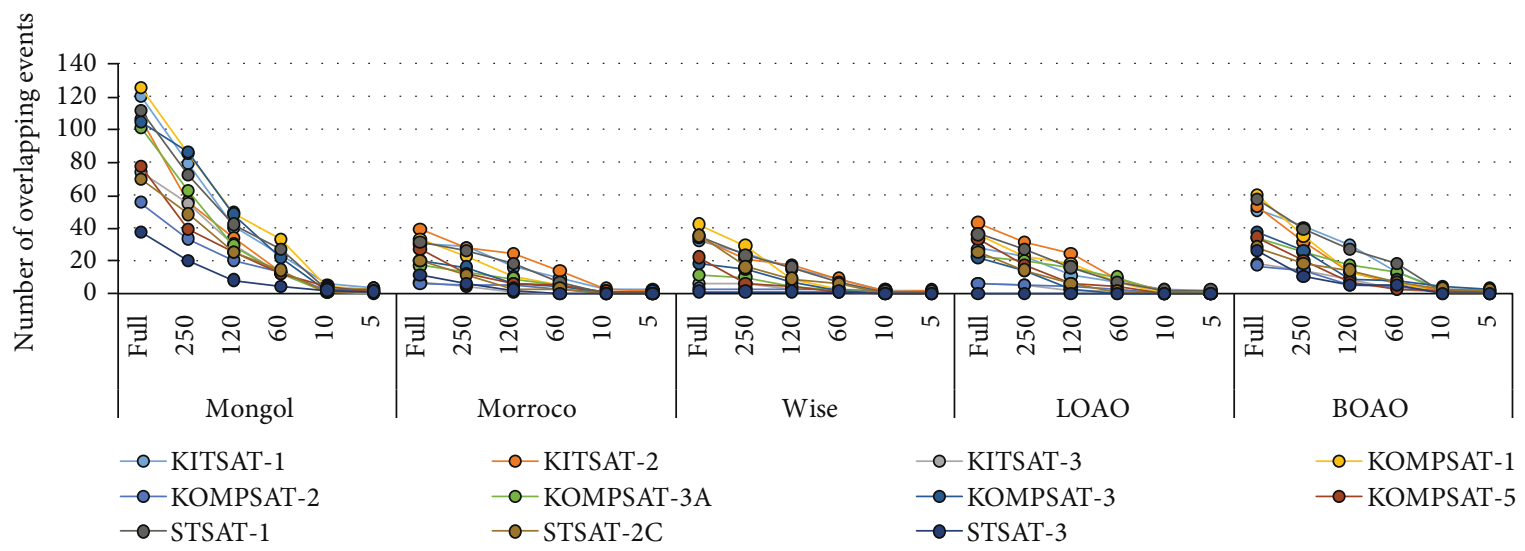

(c)

FIGURE 5: Variation of number of overlapped events for the 11 LEO observation targets for normal cases (full) and five short-arc strategies from 2014 to 2016 ((a) 2014, (b) 2015, and (c) 2016).

Mongolian site for all satellites, because it was supposed that the number of overlapped events was affected by the latitude of site and designed orbit of satellites. Except for the Mongolian site, the rest of the sites showed a similar number of overlap events. In the overall trend, almost all overlap events in a $\sim 10 \mathrm{~s}$ short-arc strategy were eliminated.

The short-arc observation strategy for the 3 selected weeks with the most overlapped events were analysed with an orbit determination test. In a short-arc observation strategy, only the arc length is considered with the same observation frequency in each observation span. The TLEs from JSpOC were used as a priori for 11 LEOs. Generally, TLE information is good to fit for the orbit of target within a few days in forward propagation [18]. However, there are sometimes abnormal TLEs for target of orbits. Therefore, we made one reference orbit for 2 weeks considering orbital information from consecutive TLEs for each observation target. This reference orbit information was used to simulate the optical observation overlapped event and to test orbit determination accuracy.

We selected five short-arc optical observation cases to compare the orbit determination effectiveness by arc length: full arc, 120,10,60, and $5 \mathrm{~s}$. The number of overlapped events was almost $70 \%$ against the full-arc case. Table 2 shows the number of overlapped events of five cases for 11 LEOs in the selected weeks of 2014, 2015, and 2016. These selected weeks experienced the most overlapping events for each year as we mentioned in Section 2.2. In the case of the selected week in 2014, there were no observation opportunities for six LEOs. However, there were the most overlapping events 
TABle 2: Number of overlapped events for 11 domestic LEO satellites at the five OWL-Net stations during the selected week for orbit determination test by the number of weekly optical observation overlapped events (2014: 4th week; 2015: 34th week; and 2016: 18th week).

\begin{tabular}{|c|c|c|c|c|c|c|c|c|}
\hline \multirow{2}{*}{ Satellite } & \multirow{2}{*}{ Year } & \multirow{2}{*}{ Total number of accesses } & \multicolumn{6}{|c|}{$\begin{array}{c}\text { Number of overlapped events for short-arc optical } \\
\text { observation strategy }\end{array}$} \\
\hline & & & Full-arc & 250 & 120 & 60 & 10 & 5 \\
\hline \multirow{3}{*}{ KITSAT-1 } & 2014 & 160 & 18 & 12 & 6 & 2 & 0 & 0 \\
\hline & 2015 & 114 & 6 & 9 & 6 & 1 & 0 & 0 \\
\hline & 2016 & 106 & 16 & 12 & 3 & 0 & 0 & 0 \\
\hline \multirow{3}{*}{ KITSAT-2 } & 2014 & 0 & 0 & 0 & 0 & 0 & 0 & 0 \\
\hline & 2015 & 63 & 11 & 7 & 4 & 1 & 0 & 0 \\
\hline & 2016 & 64 & 17 & 8 & 5 & 1 & 0 & 0 \\
\hline \multirow{3}{*}{ KITSAT-3 } & 2014 & 0 & 0 & 0 & 0 & 0 & 0 & 0 \\
\hline & 2015 & 20 & 6 & 2 & 2 & 2 & 0 & 0 \\
\hline & 2016 & 42 & 2 & 2 & 1 & 1 & 0 & 0 \\
\hline \multirow{3}{*}{ KOMPSAT-1 } & 2014 & 100 & 39 & 25 & 9 & 4 & 1 & 0 \\
\hline & 2015 & 50 & 19 & 4 & 1 & 1 & 0 & 0 \\
\hline & 2016 & 56 & 14 & 7 & 5 & 3 & 0 & 0 \\
\hline \multirow{3}{*}{ KOMPSAT-2 } & 2014 & 0 & 0 & 0 & 0 & 0 & 0 & 0 \\
\hline & 2015 & 21 & 5 & 2 & 2 & 1 & 0 & 0 \\
\hline & 2016 & 36 & 2 & 1 & 0 & 0 & 0 & 0 \\
\hline \multirow{3}{*}{ KOMPSAT-3 } & 2014 & 0 & 0 & 0 & 0 & 0 & 0 & 0 \\
\hline & 2015 & 37 & 5 & 4 & 3 & 1 & 0 & 0 \\
\hline & 2016 & 56 & 18 & 13 & 6 & 3 & 0 & 0 \\
\hline \multirow{3}{*}{ KOMPSAT-3A } & 2014 & 0 & 0 & 0 & 0 & 0 & 0 & 0 \\
\hline & 2015 & 15 & 4 & 3 & 2 & 2 & 0 & 0 \\
\hline & 2016 & 33 & 9 & 8 & 4 & 3 & 1 & 1 \\
\hline \multirow{3}{*}{ KOMPSAT-5 } & 2014 & 104 & 22 & 14 & 7 & 4 & 1 & 1 \\
\hline & 2015 & 32 & 8 & 4 & 2 & 0 & 0 & 0 \\
\hline & 2016 & 20 & 3 & 3 & 2 & 2 & 0 & 0 \\
\hline \multirow{3}{*}{ STSAT-1 } & 2014 & 100 & 42 & 25 & 13 & 6 & 0 & 0 \\
\hline & 2015 & 62 & 24 & 12 & 3 & 2 & 0 & 0 \\
\hline & 2016 & 60 & 18 & 10 & 8 & 3 & 0 & 0 \\
\hline \multirow{3}{*}{ STSAT-2C } & 2014 & 24 & 6 & 5 & 1 & 1 & 0 & 0 \\
\hline & 2015 & 64 & 35 & 27 & 8 & 3 & 0 & 0 \\
\hline & 2016 & 50 & 17 & 8 & 6 & 4 & 1 & 0 \\
\hline \multirow{3}{*}{ STSAT-3 } & 2014 & 0 & 0 & 0 & 0 & 0 & 0 & $\overline{0}$ \\
\hline & 2015 & 36 & 9 & 5 & 3 & 1 & 0 & 0 \\
\hline & 2016 & 53 & 0 & 0 & 0 & 0 & 0 & 0 \\
\hline
\end{tabular}

in the fourth week in 2014, with figures similar to those in 2015 and 2016. As we confirmed earlier in Figure 5, there were a few overlapped events for $10 \mathrm{~s}$ and $5 \mathrm{~s}$ in those weeks. In this most overlapped event week, almost all overlapped events in a $<10 \mathrm{~s}$ short-arc optical observation strategy were also eliminated like we have shown in yearly analyses plotted in Figure 5.

3.2. Orbit Determination Result for Five Short-Arc Optical Observation Strategies. The orbit determination test with the short-arc observation simulation was implemented. TLE was used as the a priori of the reference orbit as we described in the previous section. Simulated optical observation data with various arc lengths were used with assumed seeing errors of 5 arc-seconds for the right ascension and declination, respectively. In case of the tracking for LEO satellites, the time synchronization error is the dominant source of error. We assumed that the overall errors were included in that 5 arc-seconds. In the system requirements of the OWL-Net, the maximum astrometric error was assumed as 5 arc-seconds. We considered error modelling for the atmospheric density, solar radiation pressure, measurement biases, process noise, and measurement white noise. Although OWL-Net telescope can make tens of observation 
TABle 3: Orbit estimator setup and used perturbations.

\begin{tabular}{lcc}
\hline & Gravity & EGM2008 $(70 \times 70)$ \\
Perturbation & Third body & Solid tide \\
& Drag & JB2008, spherical shape \\
& SRP & Spherical shape \\
\hline \multirow{3}{*}{ Estimator } & Integrator & RKF7 $(8)$ \\
& Filter & Kalman filter \\
& Initial uncertainty & $\begin{array}{c}\text { Range: } 50 \text { m in-track: } 100 \mathrm{~m} \\
\text { cross-track: } 20 \mathrm{~m}\end{array}$ \\
\hline
\end{tabular}

points per second by using the chopper system, we deliberately simulated one point per second interval observations to check the effect of the arc length and the number of arcs. The estimator setup is summarised in Table 3. The commercial software System Tool Kit and Orbit Determination Tool Kit by Analytical Graphics Inc. were used for simulation and orbit estimation. The initial uncertainty in Table 3 meant initial covariance for sequential filtering.

The orbit determination results with observation data for seven days were compared with the propagated orbit for seven days after the end of the epoch of filtering. The results were analysed in the radial, in-track, and cross-track frame (RIC frame). Among those, radial and cross-track position uncertainty and differences from reference orbit showed a small and similar tendency for all cases. Therefore, we compared only in-track position uncertainty and difference. Figure 6 shows one-sigma position uncertainty in meters from the orbit estimation results versus the number of arcs for selected weeks in 2014, 2015, and 2016. The position uncertainty was displayed versus the total number of arcs without distinction of targets or year. Understandably, the position uncertainty was inversely proportional to the arc length for each satellite. However, the position uncertainties of $5 \mathrm{~s}$ and $10 \mathrm{~s}$ were similar to each other for all cases of each satellite. It indicates that frequent short-arc observation is more effective in maintaining the precision of the orbit than sparse longer-arc observation is.

We summarise the in-track direction orbit determination comparison results for seven days after the orbit determination in Figure 7. The estimated orbit was propagated seven days and compared with the reference orbit. The goal of the OWL-Net was maintaining orbit ephemeris with seven days to support follow-up tracking. Therefore, the maximum intrack difference is as shown in Figure 7. Even if the arc length was reduced to $5 \mathrm{~s}$ for each pass, the orbit estimation results show similar results to those of the original, full-arc case for the next seven days. In the case of STSAT-2C, however, the estimated orbit showed large differences (tens of kilometers or more). Due to its elliptical orbit, STSAT-2C has more geometrical limitations on observation opportunities. Therefore, the observation chances were more frequent when the satellite is near the apogee. The number of observation chances was similar with other satellites; the orbit determination of STSAT-2C failed in this simulation. Use of single- or double-station observations can also lead to greater

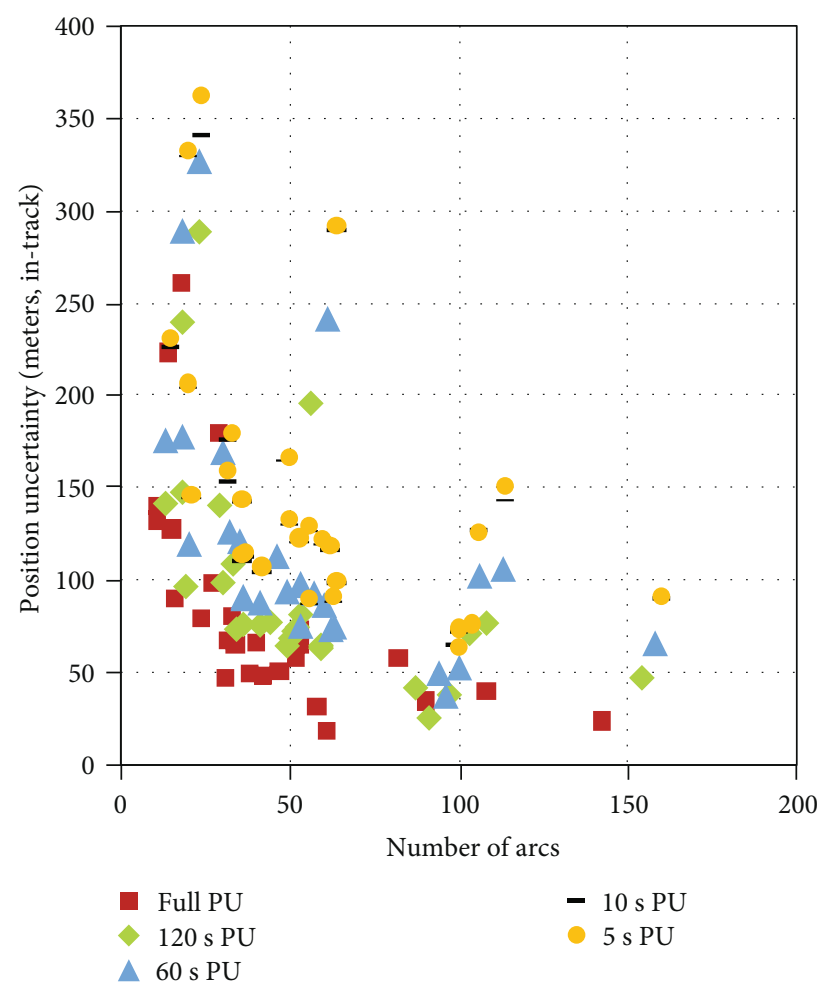

FIGURE 6: Final in-track direction position uncertainty versus the number of arcs from 7-day estimation for 11 LEOs on the most overlapped event week.

differences for the orbit estimation. We could not find any tendency of the orbit estimation accuracy between the arc length and in-track difference. This means short-arc optical observation strategy can make the orbit estimation of similar accuracy against full-arc optical observation. It can also provide more observation opportunities to many observation targets.

We confirmed that the short-arc optical observation strategy was more effective than the full-arc optical observation strategy with similar orbit accuracy and more observation opportunities. In the case of real observing conditions, the short-arc observation strategy provides a higher possibility of successful observation when weather conditions or other observation conditions like hardware maintenance are taken into account. Using short arcs, it is sometimes possible to get one or two successful shots for multishots. When using a CCD (charge-coupled device), the short-arc observing strategy also allows for a signal read-out and telescope positioning to the target. Therefore, we propose the short-arc strategy with $60 \mathrm{~s}$ arc-length as most effective optical observation tasking for the OWL-Net.

3.3. Orbit Determination Result for Five Short-Arc Optical Observation Strategies Using the Real OWL-Net Observation Data. We validated the short-arc optical observation strategy with the real observation data from the OWL-Net. The observation condition for LEO target varies with path direction, range, and track duration. We could not consider the 


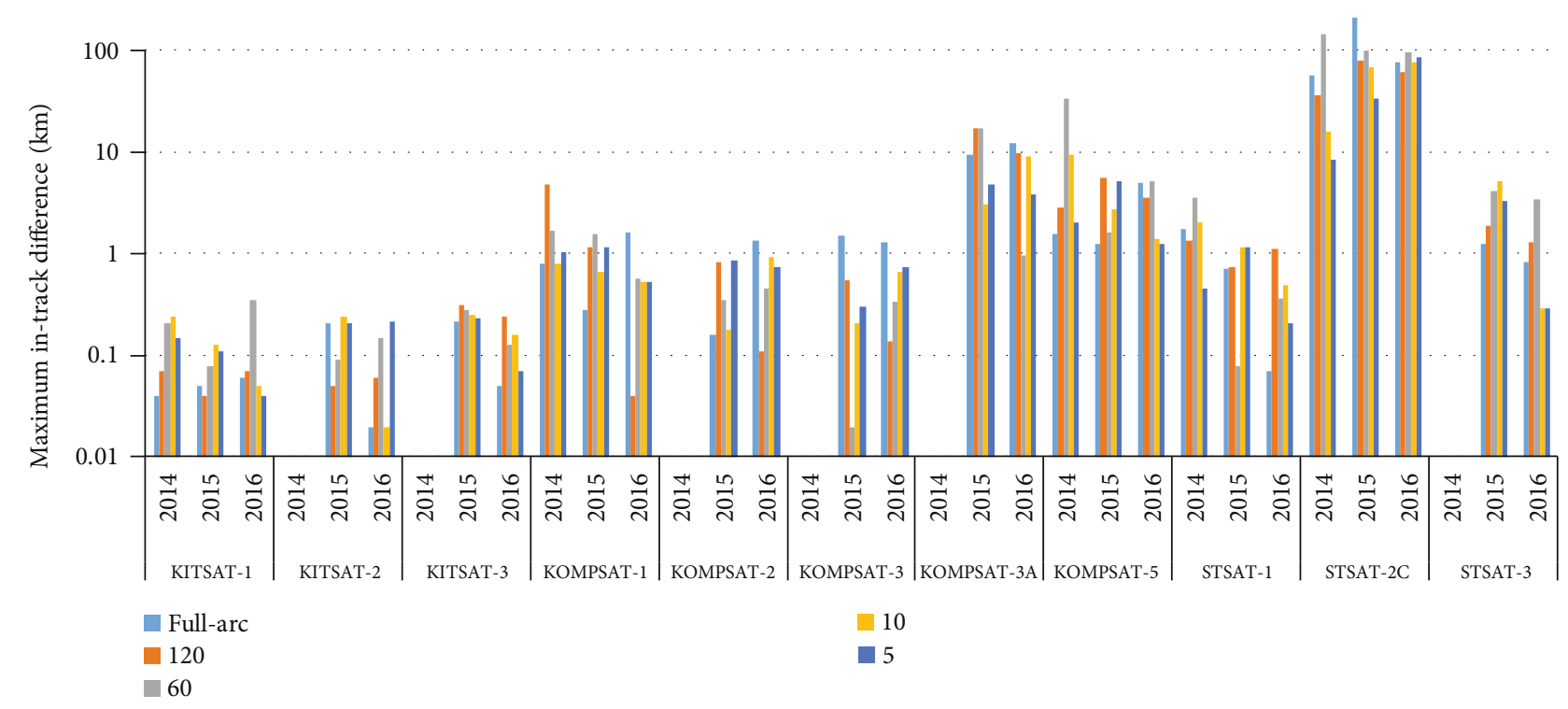

Figure 7: Maximum in-track direction difference in kilometers between the estimated orbit and the reference orbit during seven days after the end of orbit estimation with 5 optical observation test cases for seven days.

TABLE 4: Observation summary of KOMPSAT-1 from OWL-Net.

\begin{tabular}{lcccc}
\hline Site & Date & Shot & $\begin{array}{c}\text { Number of } \\
\text { points }\end{array}$ & $\begin{array}{c}\text { Arc-length } \\
\text { (seconds) }\end{array}$ \\
\hline Wise & $2017-06-23$ & 7 & 566 & 145 \\
Wise & $2017-06-25$ & 5 & 301 & 170 \\
Wise & $2017-06-26$ & 7 & 500 & 130 \\
Wise & $2017-06-29$ & 8 & 729 & 220 \\
\hline
\end{tabular}

same observation condition with the previous simulation for multi-LEO targets. Therefore, we performed the orbit determination test with the real observation data for one target with various arc-lengths.

The target satellite was KOMPSAT-1. KOMPSAT-1 is a nonoperational satellite with the altitude of $660 \mathrm{~km}$ above the ground. We observed KOMPSAT-1 for four days from 23rd to 29th of June of 2017 at the Wise observatory of the OWL-Net. The observation summary is described in Table 4 . We collected almost 2000 points with a high-speed chopping system [6]. The orbit estimation test was done for full-arc, $120 \mathrm{~s}, 60 \mathrm{~s}$, and $5 \mathrm{~s}$ cases. Figure 8 shows the comparison results of the estimated orbit and consecutive TLEs. The propagated orbit for seven days was also compared with TLEs. The differences of the four cases were very similar to each other except the $5 \mathrm{~s}$ case. Radial and cross-track differences were smaller and stable than the in-track direction fluctuation. The radial and cross-track differences were maintained within $1 \mathrm{~km}$ for duration of the estimation and the prediction. The differences for the radial and crosstrack directions in root mean square (RMS) were maintained within $0.1 \mathrm{~km}$ and $0.2 \mathrm{~km}$ for 4 cases, respectively. The intrack direction fluctuation within $3 \mathrm{~km}$ came from uncertainty of TLE data. The $5 \mathrm{~s}$ case only showed a slightly bigger difference, but still the maximum difference did not exceed
$2 \mathrm{~km}$ for 4 cases. In case of the differences for the in-track direction in RMS, the full-arc, $120 \mathrm{sec}$, and $60 \mathrm{sec}$ case errors were under $0.66 \mathrm{~m}$. And the difference of the in-track direction in RMS for the $5 \mathrm{sec}$ case was $0.67 \mathrm{~m}$. We confirmed that a $60 \mathrm{~s}$ short-arc observation is enough to maintain the orbit information stably in real observation cases.

\section{Summary and Discussion}

We analysed the effectiveness of a short-arc optical observation strategy for orbit determination using the OWL-Net. In the case of the LEO satellites, the optical observation time is limited to dawn and dusk. 11 South Korean LEO satellites are the main target of the observation of the OWL-Net. The OWL-Net is aimed at maintaining the orbital elements with weekly based orbit determination. However, the observation schedule for each target can overlap occasionally. The schedule overlapped event can result in bad quality of orbit determination with limited optical observation chances. Therefore, an effective observation strategy is required for an optical tracking system with multiple targets.

The number of weekly base optical observation overlapping events was analysed for the observation targets of the OWL-Net in 2014, 2015, and 2016. In 2015, the number of optical observation overlap events peaked in the 34th week. The 4th and 18th weeks experienced the most overlapping events of 2014 and 2016, respectively. The optical observation overlap events occurred during more than $10 \%$ of total observation opportunities for the five sites but did not show any obvious weekly trend. We simulated observation data during these 3 selected weeks in 2014,2015 , and 2016. Short-arc optical observation data were simulated for $250,120,10$, and $5 \mathrm{~s}$ of observation pass duration. In case of $10 \mathrm{~s}$ and $5 \mathrm{~s}$, only a few overlapping events remained. 


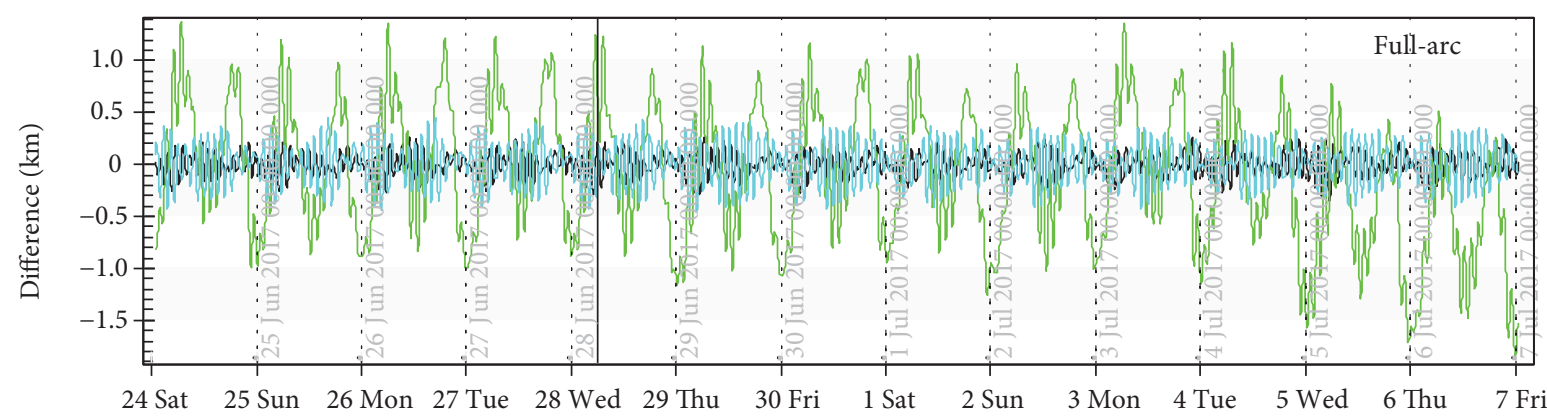
2017 Jun

(UTCG)

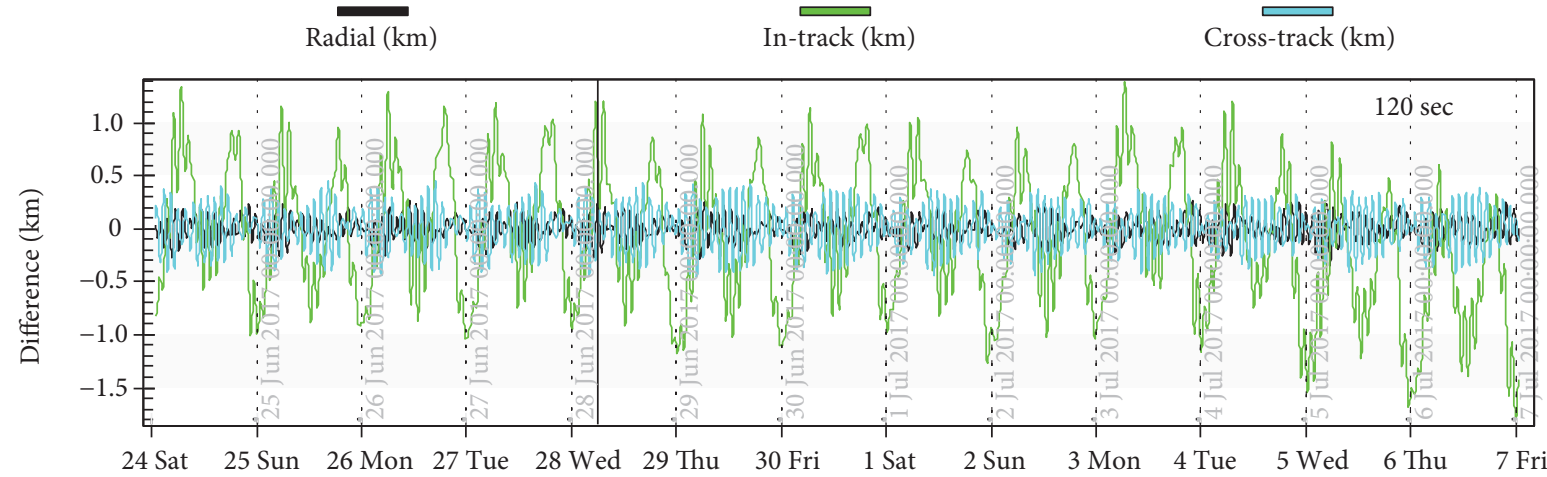

2017 Jun

(UTCG)

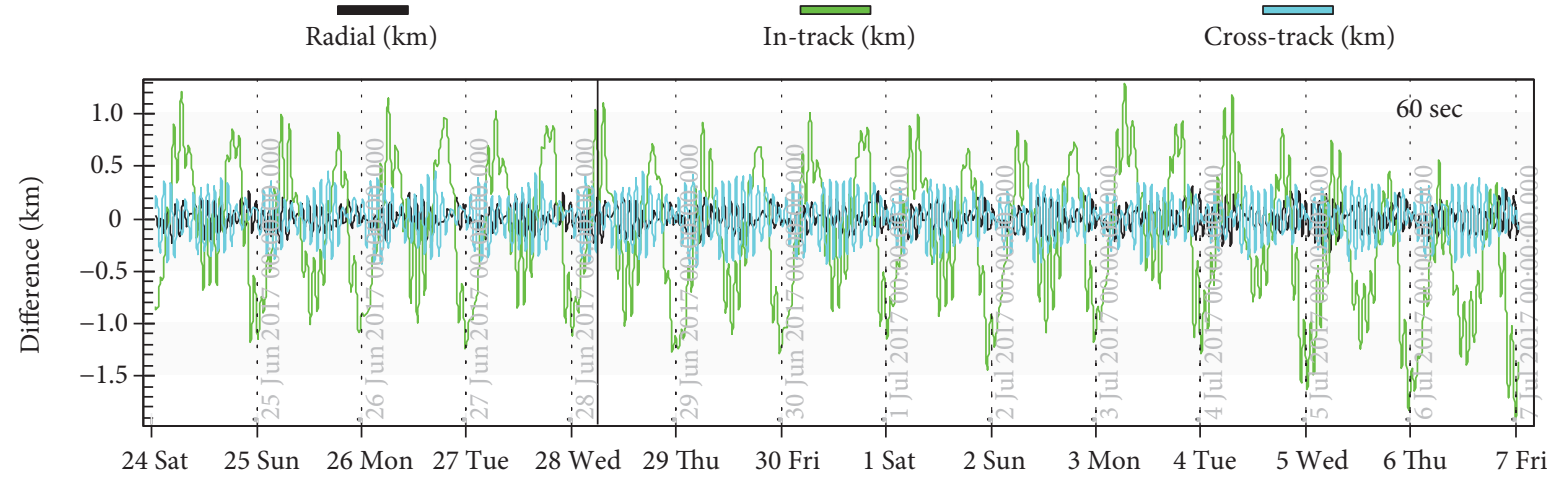

2017 Jun

(UTCG)
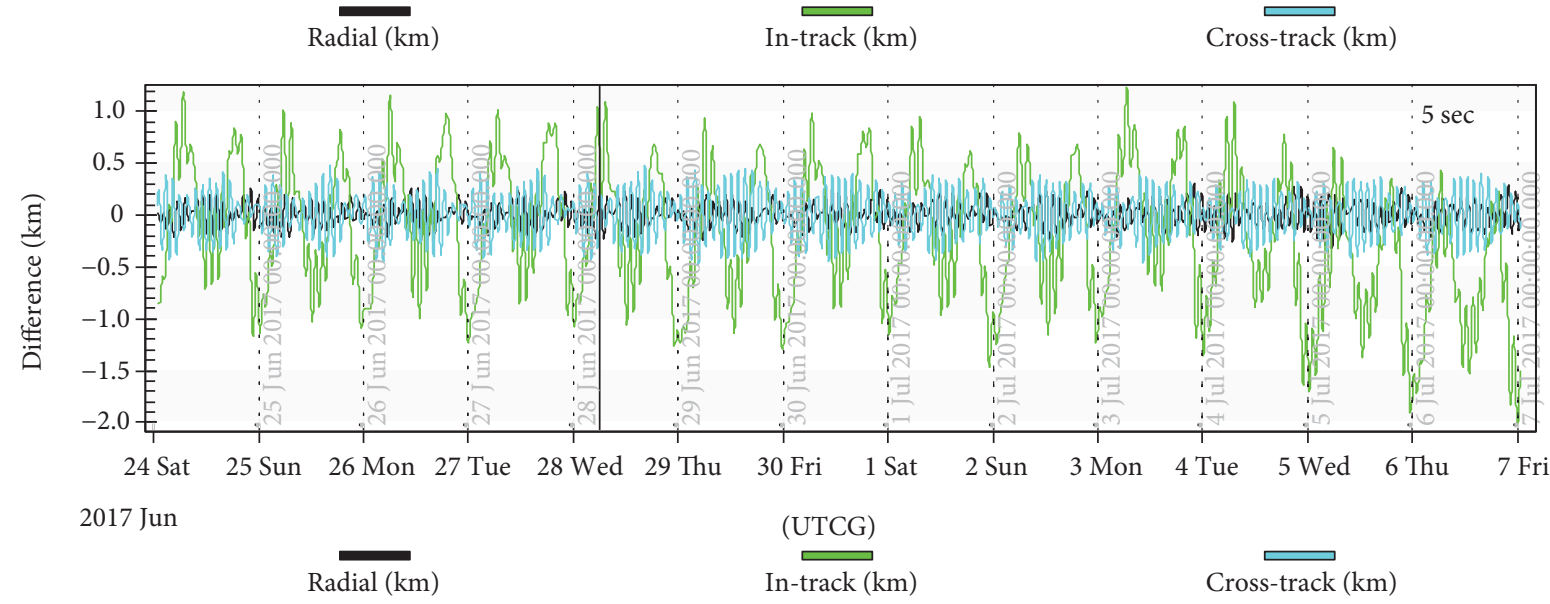

FIgURE 8: Comparison on the RIC frame with estimated orbit and consecutive TLEs for full-arc, $120 \mathrm{~s}, 60 \mathrm{~s}$, and $5 \mathrm{~s}$ cases (first seven days: estimated orbit, last seven days: propagated orbit). 
The short-arc optical observation cases show similar orbit determination results with more observation opportunities. The estimated orbits were compared with reference orbits generated by using TLE for the given target. The intrack direction orbital differences were similar to each other without any clear trends. In the case of STSAT-2C, the orbit determination test failed due to the geometrical condition of the optical observation. This result from simulation was also confirmed with orbit estimation results for KOMPSAT-1 using the real observation data from OWL-Net. We proposed $60 \mathrm{~s}$ arc-length short-arc observation strategy for scheduling strategy of real observation as seen at Section 3.3.

In this study, we have not incorporated weather conditions or the possibility of system anomalies. In a real situation, the observation opportunities can be reduced by these environmental conditions. Furthermore, the number of target satellites tracked by the OWL-Net system will increase consistently in the years to come, and growth in the number of observation targets will increase the number of overlap events. As we demonstrated in our work, the short-arc optical observation strategy can offer greater observation opportunities with similar orbit determination accuracy. Therefore, the short-arc observation strategy should be taken into account for the effective optical tracking operation of the OWL-Net.

\section{Data Availability}

The [TLE] data used to support the finding of this study are available on the website, http://www.space-track.org/. The [real optical observation data of the OWL-Net] data used to support the finding of this study have not been made available [policy of institute; Korean Astronomy and Space Science Institute].

\section{Conflicts of Interest}

The authors declare that there is no conflict of interest regarding the publication of this paper.

\section{Acknowledgments}

This study was supported by the Korea Astronomy and Space Science Institute (KASI) with the research project "Development of Space Situational Awareness Technology." The authors express their sincere appreciation to colleagues for the observation of the OWL-Net and support for this research: Myung-Jin Kim, Dong-Goo Roh, Sooyoung Kim, Aeri Kim, and Jang-Hyun Park.

\section{References}

[1] G. Veis, "Optical tracking of artificial satellites," Space Science Reviews, vol. 2, no. 2, pp. 250-296, 1963.

[2] "IADC Space Debris Mitigation Guidelines," IADC-02-01 Revision 1, 2007, http://www.iadc-online.org/Documents/.

[3] A. G. Massevitch and A. M. Losinsky, "Photographic tracking of artificial satellites," Space Science Reviews, vol. 11, no. 2-3, pp. 308-340, 1970.
[4] M. Shoemaker and L. Shroyer, "Historical trends in groundbased optical space surveillance system design," in Proceedings of the Advanced Maui Optical and Space Surveillance Technologies Conference, Wailea, HI, USA, 2007.

[5] J.-H. Kim, J.-H. Jo, J. Choi et al., "Visibility analysis of domestic satellites on proposed ground sites for optical surveillance," Journal of Astronomy and Space Sciences, vol. 28, no. 4, pp. 319-332, 2011.

[6] S.-Y. Park, K.-H. Keum, S.-W. Lee et al., "Development of a data reduction algorithm for Optical Wide Field Patrol," Journal of Astronomy and Space Sciences, vol. 30, no. 3, pp. 193206, 2013.

[7] S.-Y. Park, J. Choi, D.-G. Roh et al., "Development of a data reduction algorithm for optical wide field patrol (OWL) II: improving measurement of lengths of detected streaks," Journal of Astronomy and Space Sciences, vol. 33, no. 3, pp. 221-227, 2016.

[8] J. H. Jo, J. Choi, M. Park et al., "Early CAL/VAL process for an optical tracking system by Korea," in Proceedings of the Advanced Maui Optical and Space Surveillance Technologies Conference, Wailea, HI, USA, 2015.

[9] M. Park, J. H. Jo, S. Cho et al., "Minimum number of observation points for LEO satellite orbit estimation by OWL network," Journal of Astronomy and Space Sciences, vol. 32, no. 4, pp. 357-366, 2015.

[10] J. Y. Son, J. H. Jo, J. Choi et al., "Optical orbit determination of a geosynchronous earth orbit satellite effected by baseline distances between various ground-based tracking stations II: COMS case with analysis of actual observation data," Journal of Astronomy and Space Sciences, vol. 32, no. 3, pp. 229-235, 2015.

[11] J. Choi, J. H. Jo, M.-J. Kim et al., "Determining the rotation periods of an inactive LEO satellite and the first Korean space debris on GEO, KOREASAT 1," Journal of Astronomy and Space Sciences, vol. 33, no. 2, pp. 127-135, 2016.

[12] A. Herz and F. Stoner, "SSA sensor tasking approach for improved orbit determination accuracies and more efficient use of ground assets," in Proceedings of the Advanced Maui Optical and Space Surveillance Technologies Conference, Wailea, HI, USA, 2013.

[13] D. A. Vallado and S. S. Carter, "Accurate orbit determination from short-arc dense observational data," Advances in the Astronautical Sciences, vol. 97, pp. 1587-1602, 1998.

[14] J. C. Bennett, J. Sang, C. Smith, and K. Zhang, "An analysis of very short-arc orbit determination for low-earth objects using sparse optical and laser tracking data," Advances in Space Research, vol. 55, no. 2, pp. 617-629, 2015.

[15] S. Kawase, "Orbit determination accuracy for optically tracked near-synchronous objects," in Proceedings of the International Symposium on Space Dynamics, Biarritz, France, 2000.

[16] J. Tombasco and P. Axelrad, "A study of the achievable geosynchronous angles-only orbit estimation accuracy," The Journal of the Astronautical Sciences, vol. 58, no. 2, pp. 275-290, 2011.

[17] D. A. Vallado and W. D. McClain, Fundamentals of Astrodynamics and Applications, Kluwer Academic Publisher, Boston, MA, USA, 2nd edition, 2001.

[18] D. A. Vallado, B. B. Virgili, and T. Flohrer, "Improved SSA through orbit determination of two-line element sets," in 6th European Conference on Space Debris, Darmstadt, Germany, 2013. 


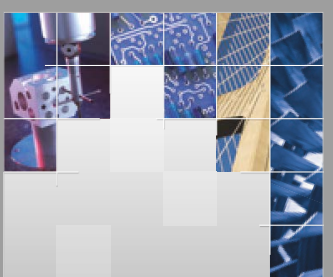

\section{Enfincering}
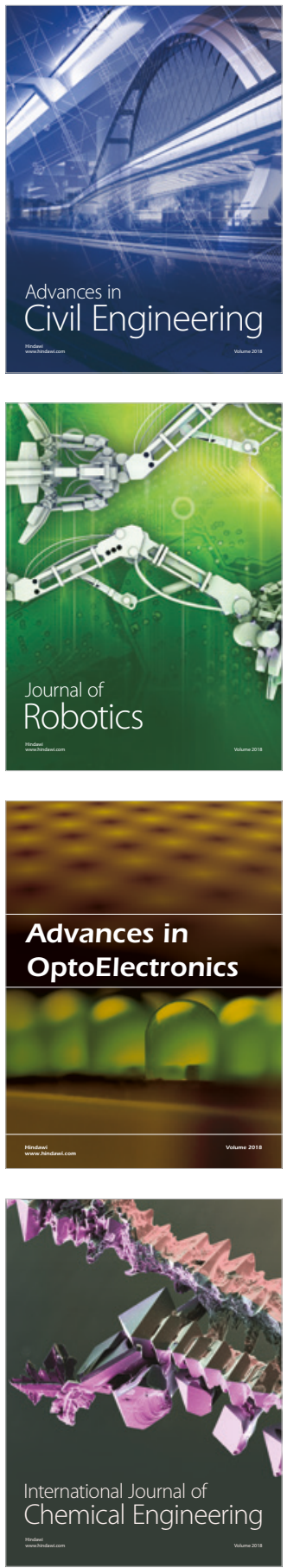

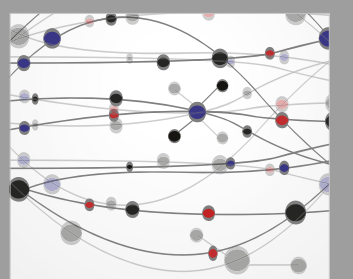

\section{Rotating \\ Machinery}

The Scientific World Journal

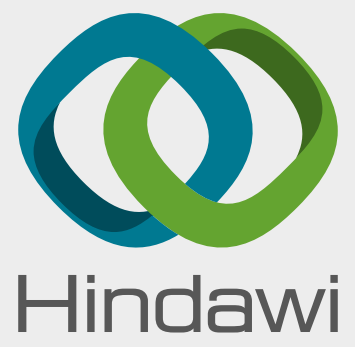

Submit your manuscripts at

www.hindawi.com
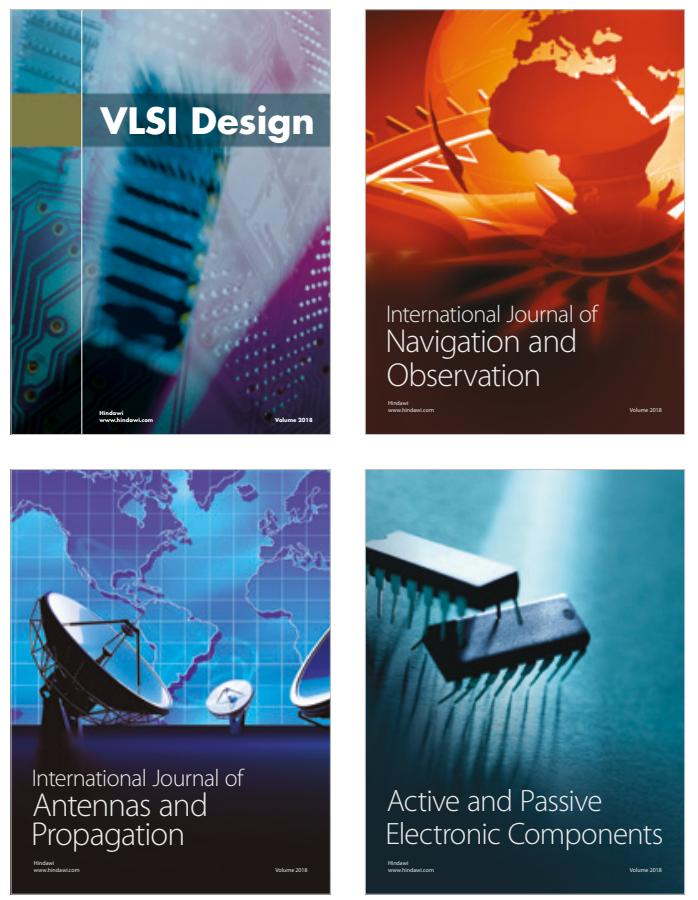
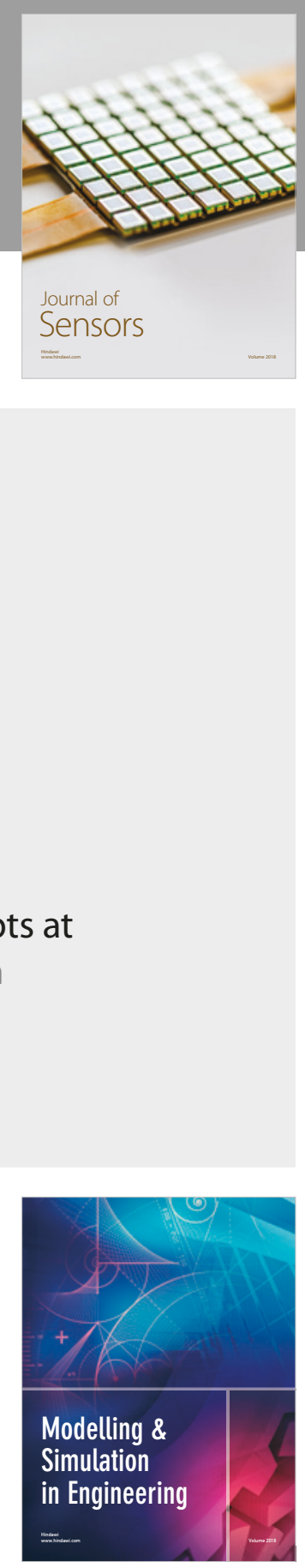

\section{Advances \\ Multimedia}
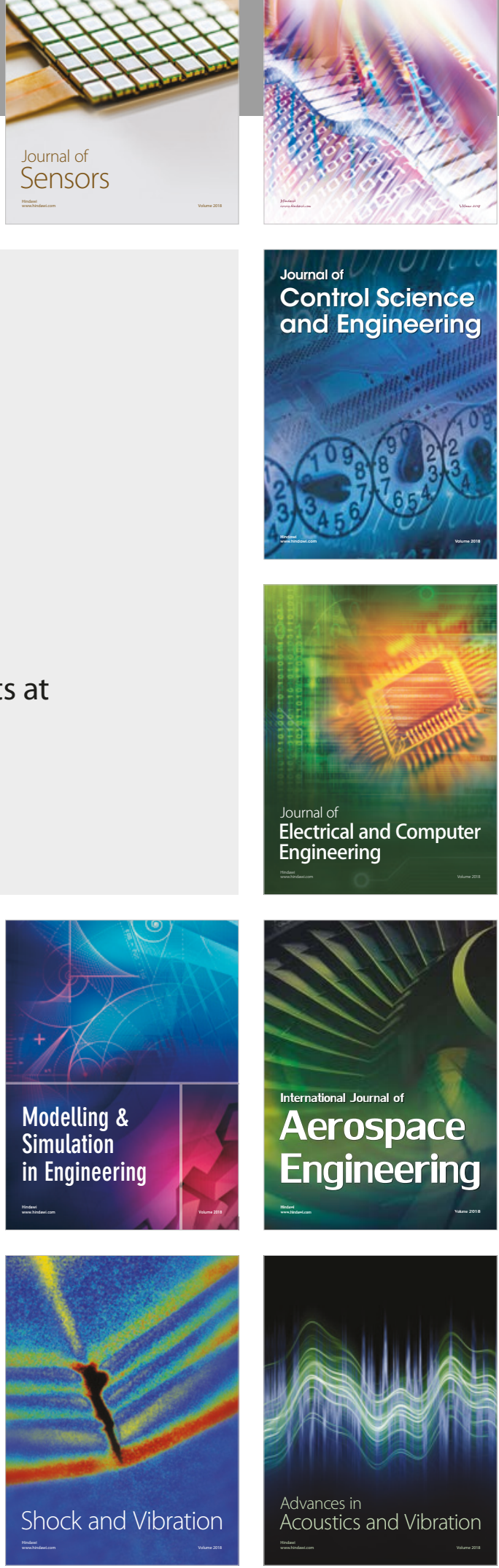